\title{
7q22 deletion in myeloid malignancies: be attentive to the design of the FISH probe
}

\author{
Délétion 7q22 dans les pathologies myéloides: \\ attention au design des sondes FISH
}

\author{
Stéphanie Struski \\ Isabelle Luquet \\ Institut universitaire du cancer Toulouse \\ - Oncopole, Toulouse, France \\ <struski.stephanie@iuct-oncopole.fr>
}

Article received December 12, 2018,

accepted January 12, 2019

Partial deletion of the long arm of chromosome 7 $[\operatorname{del}(7 q), 7 q-]$ is a highly recurrent chromosomal aberration in myelodysplastic syndrome (MDS) and acute myeloid leukemia (AML) $[1,2]$. In AML, isolated 7q- is assigned to the intermediate genetic prognostic risk group (ELN classification) or the high risk prognostic group (revised MRC classification). In MDS, isolated 7q- is classified into the intermediate cytogenetic prognostic risk group or into the poor risk group if associated with another abnormality according to the revised IPSS (International prognostic scoring system). The deleted regions are distributed along the long arm of chromosome 7 but studies have indicated the existence of at least three commonly deleted regions, at bands 7q21-22, 7q34 and 7q35-q36 [3, 4]. Our study focused on small 7q22 deletions, suspected on karyotype analysis and requiring confirmation by FISH. We explored the region using commercial and home-made probes.

17 patients with myeloid malignancies (4 AML, 10 MDS, $1 \mathrm{MPN}$ - myeloproliferative neoplasm, $2 \mathrm{MDS} / \mathrm{MPN}$ ) were selected because a small 7q22 deletion was observed on the karyotype.

The deletion was isolated in 15/17 cases and associated with trisomy 8 or 12 p deletion each in one case. According to the manufacturer's specifications, commercial 7q22 locus specific probes offer different coverage areas targeting CUX1, RELN or KMT2E genes. In our series $15 / 17$ patients showed a deletion of the $C U X 1$ probe and $2 / 17$ patients had deletion of the three loci (CUX1, RELN and

Reprints: S. Struski
$K M T 2 E$ probes). No patient had an isolated deletion of $R E L N$ and/or KMT2E. Thus 15 patients would not have been detected with commercial probes not targeting the $C U X 1$ gene. We then explored the size of this deletion centered on the CUX1 gene using different home-made probes. We studied the region between the MUC17 and KMT2E genes in band 7q22 according to Jerez et al. [3] and Honda et al. [5] (figure 1 figure 1).

The following BACs were used : RP11-1108K10 / RP11151L12 / RP5-1059M17 (covering the MUC17, TRIM56, SERPINE1, AP1S1, VGF, NAT16, MOGAT3, PLOD3, ZNHIT1, CLDN15, FIS1, IFT22, COL26A1 genes), RP11333G13 (CUX1 gene), RP4-672O11 / RP11-188E1 (RELN gene), RP11-148A10 / RP11-708P17 (BACs flanking the $K M T 2 E$ gene). The $7 \mathrm{q} 22$ deletion including the $C U X 1$ gene extended to the MUC17 gene in 13/17 patients. For the 4 other patients the deletion was limited to RP5-1059M17 and RP11-333G13 BACs (figure 1). We therefore advice to limit FISH exploration of the $7 q 22$ region with probes targeting the $C U X 1$ gene.

In most cases $7 q 22$ deletion is identified on karyotype analysis. However, in some cases, the deletion can only be suspected and for certainty additional FISH is essential. The design of commercial FISH probes is consensual for the majority of chromosome rearrangements but in the case of the -7 / 7q- syndrome, many target genes have been suggested and this has generated probes targeting the same chromosomal region but not the same genes. Target gene identification is becoming more efficient and more accurate and the design of commercial probes should be updated regularly in order to remain appropriate. 


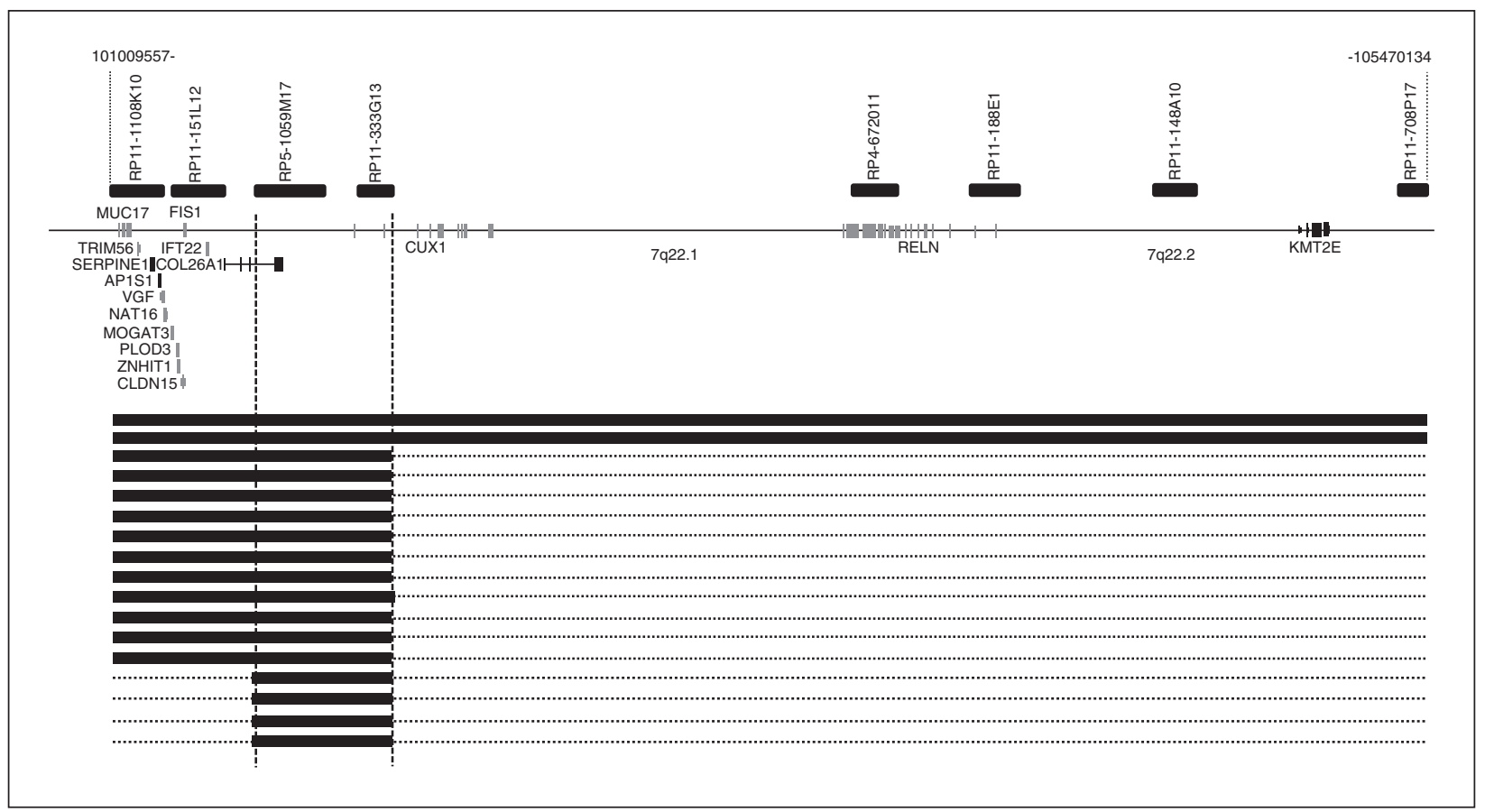

Figure 1. Identification of the common minimal deleted region. Representation of the 7q22.1 to 7q22.2 region with the BACs used. The deleted region for each of the 17 patients is indicated by a black box, the dotted line represents the conserved area. The minimal region common to these patients covers BACs RP5-1059M17 and RP11-333G13 (372 kb).

Conflict of interest: none of the authors has any conflict of interest to disclosure.

\section{References}

1. Johnson E, Cotter FE. Monosomy 7 and $7 \mathrm{q}-$ associated with myeloid malignancy. Blood Rev 1997 ; 11(1) : 46-55.

2. Hasle H, Aricò M, Basso G, Biondi A, Cantù Rajnoldi A, Creutzig $\mathrm{U}$, et al. Myelodysplastic syndrome, juvenile myelomonocytic leukemia, and acute myeloid leukemia associated with complete or partial monosomy 7. European Working Group on MDS in Childhood (EWOGMDS). Leukemia 1999; 13:376-85.

3. Jerez A, Sugimoto Y, Makishima H, Verma A, Jankowska AM, Przychodzen $\mathrm{B}$, et al. Loss of heterozygosity in 7q myeloid disorders : clinical associations and genomic pathogenesis. Blood 2012;119(25) : 6109-17.

4. Hosono N, Makishima H, Jerez A, Yoshida K, Przychodzen B, McMahon $\mathrm{S}$, et al. Recurrent genetic defects on chromosome $7 \mathrm{q}$ in myeloid neoplasms. Leukemia $2014 ; 28: 1348-51$

5. Honda $\mathrm{H}$, Nagamachi $\mathrm{A}$, Inaba T. -7/7q- syndrome in myeloid-lineage hematopoietic malignancies : attempts to understand this complex disease entity. Oncogene 2015 ; 34 : 2413-25. 